\title{
Catheter-based endobronchial electroporation is feasible for the focal treatment of peribronchial tumors
}

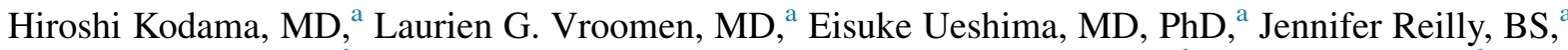 \\ Whitney Brandt, MD, ${ }^{\mathrm{b}}$ Lee-Ronn Paluch, BVSc, ${ }^{\mathrm{c}}$ Sebastien Monette, DMV, ${ }^{\mathrm{d}}$ David Jones, MD, ${ }^{\mathrm{b}}$ \\ Stephen B. Solomon, MD, ${ }^{\mathrm{a}, \mathrm{e}}$ and Govindarajan Srimathveeravalli, $\mathrm{PhD}^{\mathrm{a}, \mathrm{e}}$
}

\begin{abstract}
Objective: To evaluate the feasibility of catheter-based endobronchial electroporation for the treatment of peribronchial tumors and assess the incidence of treatment-related adverse events.
\end{abstract}

Methods: Cytotoxicity of electroporation with or without cisplatin or gefitinib was assessed in vitro with lung cancer and normal cell lines. A novel catheter was designed for endobronchial electroporation, and computer simulations were used to predict in vivo treatment effects. Electroporation with the test catheter was performed ( $2000 \mathrm{~V}, 70$ pulses) in the main bronchus of 8 pigs at 11 locations. Computed tomography imaging was performed before they were killed at 4 hours $(6$ animals $)$ or 4 weeks $(2$ animals $)$ posttreatment. Treated airway and surrounding parenchyma were compared with sham treatment via gross and histopathology.

Results: Significant cell death due to electroporation and increased cytotoxicity in combination with cisplatin or gefitinib were observed in cancer cells only $(P<.05)$. Simulations predicted penetrative electroporation of peribronchial parenchyma without tissue heating. Electric pulse delivery in vivo induced transient venous and bronchial spasms that resolved without intervention. Cross-sectional measurement of electroporation effects on computed tomography (14.4 \pm 1.4 by $10.5 \pm 1.3 \mathrm{~mm})$ and gross pathology $(17.2 \pm 3.0$ by $8.8 \pm 0.6 \mathrm{~mm})$ were representative of values predicted by simulation $(P<.001)$. Cell death due to irreversible electroporation was observed in bronchial and parenchymal tissue in acute tissue samples. Treated lung rapidly recovered from the effects of electroporation without change in bronchial patency at 4 weeks posttreatment.

Conclusions: Catheter-based endobronchial electroporation is a reproducible technique that can be used to treat peribronchial tumors in combination with cisplatin, without affecting patency of the treated bronchus. (J Thorac Cardiovasc Surg 2018;155:2150-9)

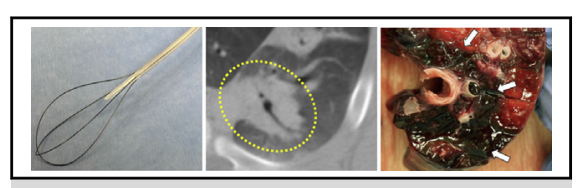

Left, Endobronchial catheter. Middle, CT image of treated lung. Right, Electroporated lung.

\section{Central Message}

Endobronchial electroporation is a feasible and reproducible technique that can be used for focal treatment of peribronchial tumors in combination with cisplatin.

\section{Perspective}

Management of malignant central airway obstruction is limited by a lack of techniques for safe but penetrative peribronchial treatment. Electroporation allows penetrative treatment of peribronchial tissue without affecting the patency of the treated bronchus. Coadministration of standard-of-care drugs such as cisplatin can further increase treatment efficacy.

See Editorial Commentary page 2160.
From the ${ }^{\mathrm{a}}$ Interventional Radiology Service, Department of Radiology and ${ }^{\mathrm{b}}$ Thoracic Service, Department of Surgery, Memorial Sloan-Kettering Cancer Center, New York; ${ }^{\mathrm{C}}$ Center of Comparative Medicine and Pathology, Memorial Sloan Kettering Cancer Center, Weill Cornell Medicine, Hospital for Special Surgery, New York; ${ }^{\mathrm{d}}$ Laboratory of Comparative Pathology, Memorial Sloan Kettering Cancer Center, The Rockefeller University, Weill Cornell Medicine, New York; and ${ }^{\mathrm{e}}$ Department of Radiology, Weill Cornell Medical College, New York, NY.

The authors acknowledge the support of the National Institutes of Health Cancer Center Support Grant (P30 CA008748) for core laboratory services that were used for the presented work. G.S. acknowledges grant support from the Society of Interventional Radiology (SIR) Foundation and the National Cancer Institute of the National Institutes of Health under Award Number U54CA137788/U54CA132378.

Received for publication May 23, 2017; revisions received Nov 9, 2017; accepted for publication Nov 16, 2017; available ahead of print Jan 19, 2018.

Address for reprints: Govindarajan Srimathveeravalli, PhD, H112, Department of Radiology, 1275 York Ave, New York, NY 10065 (E-mail: srimaths@mskcc.org). $0022-5223 / \$ 36.00$

Copyright $($ c 2017 by The American Association for Thoracic Surgery

https://doi.org/10.1016/j.jtcvs.2017.11.097
Central airway obstruction by primary or metastatic tumors can cause significant morbidity to patients, increasing the overall risk of mortality. ${ }^{1}$ Bronchoscopyguided electrocautery, ${ }^{2}$ laser, ${ }^{3-5}$ and thermal ablation ${ }^{6-8}$ have been evaluated for the debulking and focal treatment of exophytic central airway tumors with encouraging results. ${ }^{9-12}$ However, bronchoscopy-guided

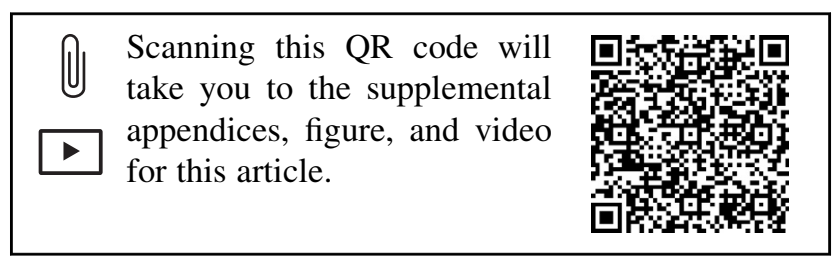



Abbreviations and Acronyms
CT = computed tomography
$\mathrm{H} \& \mathrm{E}=$ hematoxylin and eosin
HBEC = human bronchial epithelial cell line
IRE = irreversible electroporation
$\mathrm{RE}=$ reversible electroporation

ablation is considered unsuitable for the treatment of peribronchial or extrinsic tumor subtypes, and patients with such tumors are usually palliated with stent placement. $^{1,12,13}$ Stent patency is often challenged by continued tumor growth, formation of granulation tissue, and mucous blockage due to impeded clearance, necessitating repeated interventions. ${ }^{13-16}$ New ablation techniques and devices that can safely treat peribronchial tumors can improve the management of patients with central airway obstruction from malignant disease.

Permeabilization of the cell membrane with electric pulses is called electroporation, and the effects of permeabilization can either be transient (reversible electroporation $[\mathrm{RE}]$ ) or permanent (irreversible electroporation [IRE]). ${ }^{17}$ Transient permeabilization of the cell membrane during RE allows increased transport of chemotherapeutics into cancer cells, leading to tumor destruction. IRE of the cell membrane causes cell death from loss of homeostasis, and IRE has been developed for the focal ablation of tumors in the liver and pancreas. ${ }^{18}$ Electroporation in patients has also been observed to preserve the architecture and function of ductal organs such as the bile duct and large blood vessels that lie within or are adjacent to the treatment zone. ${ }^{19}$ These features make electroporation an attractive candidate for the endobronchial treatment of malignant central airway obstruction without affecting airway patency. The objective of this study was therefore to evaluate the feasibility of catheter-based endobronchial electroporation for the treatment of peribronchial tumors and to investigate the incidence of treatment-related adverse events.

\section{METHODS}

\section{In Vitro Assessment of Electroporation}

Three human non-small cell lung cancer cell lines (HCC827, A549, and H1975) and the human bronchial epithelial cell line (HBEC) were used to assess in vitro electroporation. All cells were treated with 70 pulses at varying voltages. See Appendix E1, ${ }^{20}$ for culture and treatment details. Fluorescent microscopy using acridine orange $(2 \mu \mathrm{L} / \mathrm{mL})$ and propidium iodide $(20 \mu \mathrm{L} / \mathrm{mL})$ was performed immediately after treatment to discriminate the population of nonelectroporated, electroporated, and necrotic cells. Changes in cell viability due to electroporation were assessed with an MTT assay (Invitrogen, Carlsbad, Calif) performed 4 hours after treatment and were normalized against viability of cells receiving sham treatment.

\section{In Vitro Evaluation of Electroporation in the Presence of Cisplatin or Gefitinib}

In vitro techniques described in the preceding section were used to assess outcomes when cells underwent electroporation with cisplatin or gefitinib (Santa Cruz Biotechnology, Santa Cruz, Calif) and compared with treatment with drug alone (see Appendix E1 for treatment details). ${ }^{20}$ The cells were incubated after treatment, and cell proliferation was assessed with an MTT assay at 24 or 72 hours posttreatment with cisplatin or gefitinib, respectively.

\section{Computer Simulation of In Vivo Electroporation and Ex Vivo Temperature Measurement}

Numerical models were constructed and computer simulations were performed to estimate the expected region of peribronchial lung parenchyma that would experience electroporation and identify the potential for thermal injury to the airway when using the endobronchial catheter. Appendix E2 and Figure 1 describe the design as well as the construction of the endobronchial catheter (see Video 1 for a demonstration of the working principle). Ex vivo temperature measurements were performed to record heat development during pulse delivery. For details on the numerical models and ex vivo temperature measurements, see Appendices E3 ${ }^{21,22}$ and E4.

\section{In Vivo Evaluation of Endobronchial Electroporation}

Eight male Yorkshire pigs (3-4 months old; weight range, $36-55 \mathrm{~kg}$ ) were used under an Institutional Animal Care and Use Committeeapproved protocol for this study. The animals were sedated, intubated, and underwent treatment while under inhaled anesthesia ( $2 \%-3 \%$ isoflurane in $100 \%$ oxygen). After anesthesia, the catheter electrode was placed through the intubation tube using fluoroscopy or computed tomography (CT) guidance (Figure 2, $A$ and $B$ ). The catheter was advanced to right or left mainstem bronchus, and the basket was unfurled at a location that was at least $3 \mathrm{~cm}$ from the heart. Three animals received treatments in both the left and right main bronchus, whereas the remaining 5 received treatment in only 1 bronchus, with the contralateral bronchus undergoing sham device placement. Animals received an intravenous, short-acting muscular paralytic (rocuronium, $1.2 \mathrm{mg} / \mathrm{kg}$ ) before pulse delivery to reduce neuromuscular activation from electrical stimulation. Endobronchial pulse delivery was performed with electrocardiogram cardiac gating (Nanoknife; AngioDynamics, Latham, NY) using a single treatment setting (2000 V, $90-\mu$ s pulse length at $1 \mathrm{~Hz}, 70$ pulses). CT imaging was repeated immediately after pulse delivery and at 2 hours after treatment in all animals. Six animals ( 8 treatments) were killed humanely within 4 hours of treatment and 2 animals ( 3 treatments) recovered from anesthesia and were followed for 28 days posttreatment with additional CT imaging on days 7 and 28 (see Appendix E5 for details on imaging and analysis).

\section{Gross Pathology and Histopathology}

The lungs and trachea were extracted en bloc from all animals immediately after they were killed for gross pathology and histopathology via hematoxylin and eosin (H\&E), terminal deoxynucleotidyl transferasemediated dUTP nick end labeling (cell death), and anticleaved caspase-3 (cell apoptosis) (see Appendix E6 for information on tissue processing and histopathology).

\section{Statistical Analysis}

Statistical analyses were conducted with EZR software (Saitama Medical Center, Jichi Medical University, Tochigi, Japan) or STATA (StataCorp, College Station, Tex). $P<.05$ was inferred as an indication of a significant difference in measurements (see Appendix E7 for information on statistical techniques and analysis). 


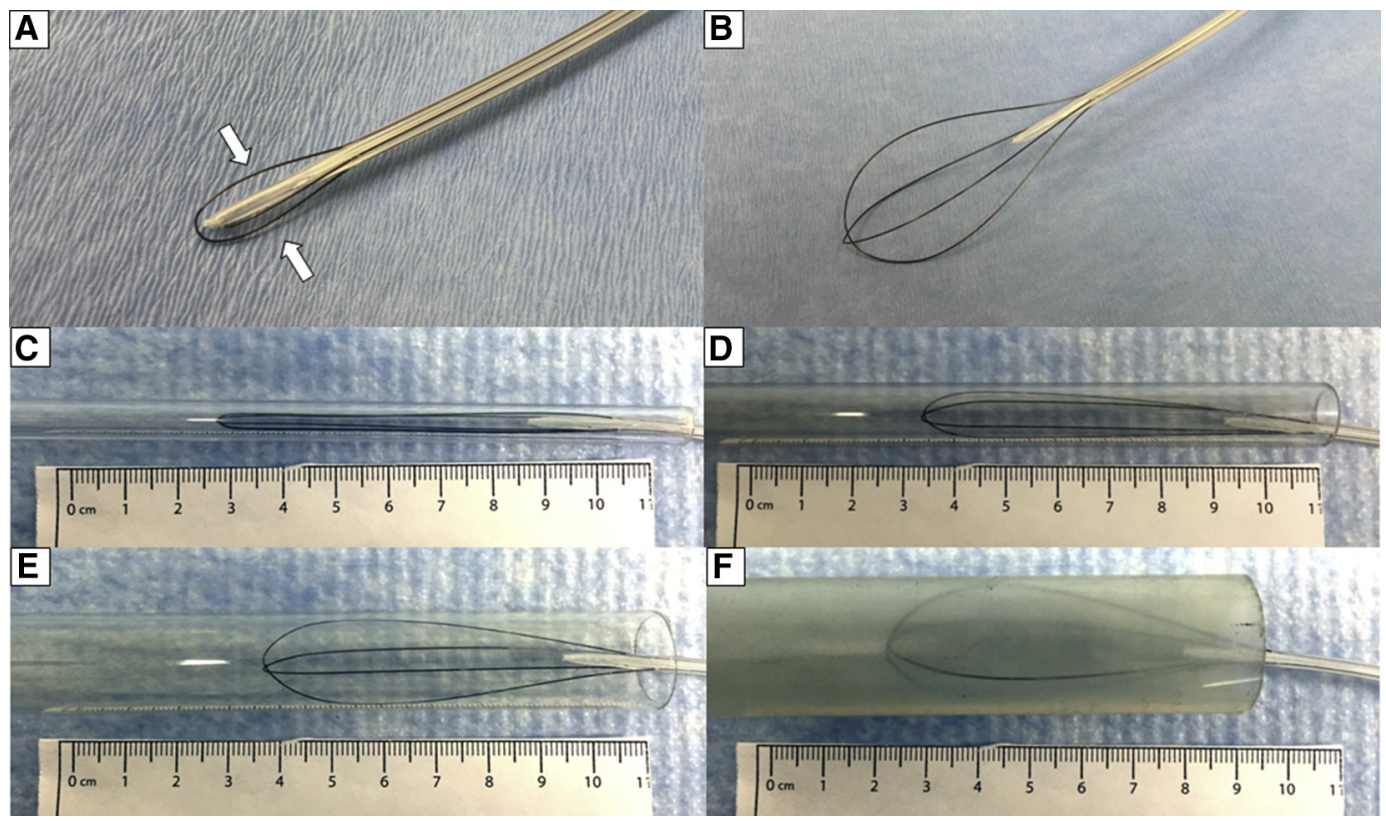

FIGURE 1. Endobronchial catheter with basket electrode used for performing electroporation. A-B, A basket electrode was constructed at the tip of a multilumen catheter such that insert-withdrawing the electrode from the distal tip expanded or collapsed the basket. This basket configuration provided uniform surface contact with lumen of varying diameters (C: $5 \mathrm{~mm}, \mathrm{D}: 10 \mathrm{~mm}, \mathrm{E}: 17 \mathrm{~mm}$, and F: $21 \mathrm{~mm}$ ).

\section{RESULTS}

\section{Malignant Cells are More Sensitive to the Effects of Electroporation}

Electroporation was detected in both normal and cancer cells even at the lowest tested voltage $(500 \mathrm{~V})$, where greater percentage of HBEC cells underwent electroporation when compared with cancer cells. The percentage population of HBEC cells undergoing electroporation saturated at greater voltages, whereas the percentage of cancer cells undergoing electroporation increased in a dose-dependent fashion in proportion to the applied voltage. The greatest

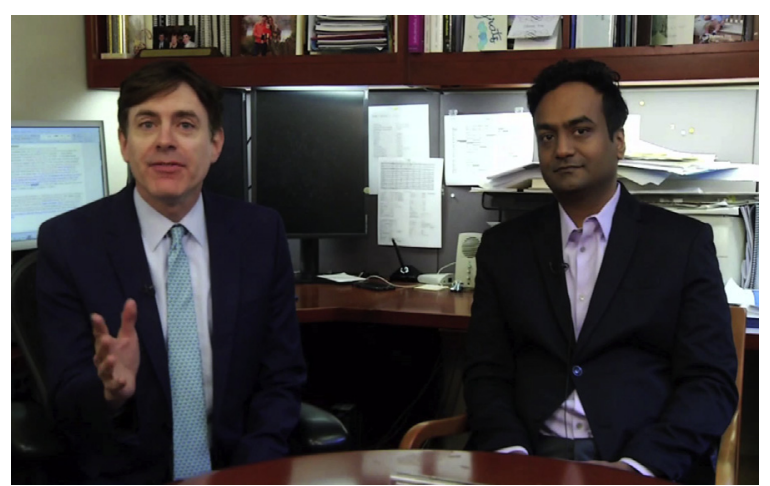

VIDEO 1. Authors describing the principles of IRE and demonstrating the device. The corresponding author (G.S) and key author (S.B.S) describe the technique of IRE and its specific benefits and demonstrate the working principle of the device that allows targeting of airway of varying sizes. IRE, Irreversible electroporation. Video available at: http://www.jtcvsonline.org/ article/S0022-5223(17)33003-9/fulltext. test voltage $(2000 \mathrm{~V})$ induced RE or IRE in $>80 \%$ of the population for all cancer cell lines (Figure 3, B-D). HBEC cells seemed more resilient to cell death from IRE, and the H1975 cells were most sensitive to IRE. Cell death was observed at increased numbers in the immediate vicinity of the electrodes, with reduced incidence between the electrodes and the periphery of the treatment zone where reduced electric field strength was expected (Figure 3, $E$ and $F$ ). Pulse voltage of $1000 \mathrm{~V}$ resulted in RE or IRE of at least $50 \%$ of cells in all cell lines and was presumed to be the $\mathrm{IC}_{50}$ value for lung electroporation. At 4 hours posttreatment, electroporation had the least impact on the proliferation and cell viability of HBEC cells, with slight reduction in proliferation only in the $2000 \mathrm{~V}$ setting (Figure 3, G-J). Cancer cell lines exhibited reduction in proliferation in proportion to the applied voltage, with approximately $50 \%$ and $75 \%$ reduction in numbers when treated with 1500 and $2000 \mathrm{~V}$ pulses, respectively.

\section{Electroporation Enhances the Cytotoxicity of Cisplatin and Gefitinib in Cancer Cells}

Compared with treatment with cisplatin or gefitinib alone, electroporation in the presence of either drug significantly reduced cell proliferation in all cancer cell lines (all $P<.0125$ ) (Figure 4). The minimal required voltage to achieve a significant reduction in cell proliferation differed among the cancer cell lines. The HCC827 cell line exhibited the greatest reduction in proliferation after combination treatment with electroporation and cisplatin, followed by 


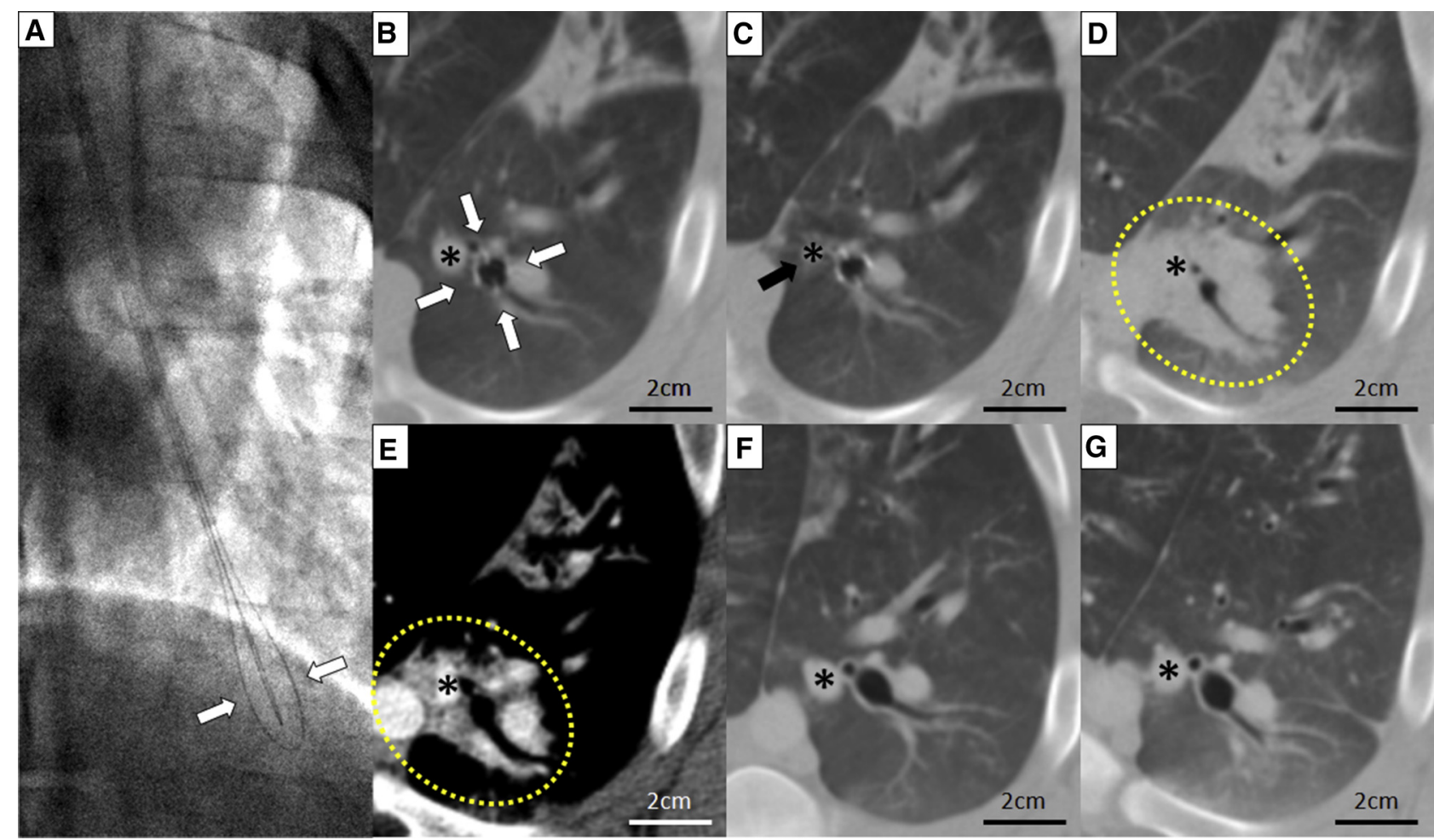

FIGURE 2. Intraprocedural imaging during endobronchial electroporation and follow-up CT imaging of treated lung. A, The basket electrode was guided into the mainstem bronchus under fluoroscopy guidance and unfurled. B, Pretreatment CT imaging demonstrating uniform electrode contact with bronchial wall (arrows); pulmonary vein adjacent to the bronchus is marked with an asterisk. C, There is obvious spasm of the vein (arrow and asterisk) immediately after pulse delivery. D, Electroporated lung appears as a region of dense consolidation (bound by dotted yellow line). Asterisk indicates the vein. E, Contrast-enhanced CT images show patent vein (asterisk) and the absence of thrombus formation. Electroporated lung appears as a region of dense consolidation (dotted yellow circle). F, Day 7 imaging shows resolution of treatment related consolidation of the lung. The diameters of pulmonary vein and treated bronchus are similar to baseline. Asterisk indicates the vein. G, Day 28 imaging shows normal lung tissue with no obvious signs of treatment effect, scar formation, or granulation tissue in the treated bronchus. Asterisk indicates the vein.

A549 and H1975 cells, whereas HBEC proved resilient to such treatment (Figure 4). Compared with electroporation alone, combination treatment produced significantly greater reduction in proliferation at lower voltages $(500$ and $1000 \mathrm{~V}, P<.0125)$, but the difference was not significant at greater voltages $(1500$ and $2000 \mathrm{~V})$. The antiproliferative effect of gefitinib was greatest in HCC827 (Figure 5) and the effect was modest in the other cancer cell lines (A549 and H1975).

\section{Numerical Simulations can Estimate Regions of Electroporation After Endobronchial Pulse Delivery}

Computer simulations generated the expected electric field distribution in the lung during endobronchial electric pulse delivery with the basket electrode. Pulse delivery at $2000 \mathrm{~V}$ provided maximum penetration of the electric field within the peribronchial lung parenchyma without causing clinically significant increase in tissue temperature. Electric fields sufficient to induce electroporation in vitro (500-2000 $\mathrm{V} / \mathrm{cm}$ ) were predicted in a $1-\mathrm{cm}$ circumferential region surrounding the basket electrode (Figure 6, A). Large blood vessels and airways were observed to affect the electric field distribution, with reduction of the electric field strength below thresholds for electroporation at microscopic regions in the immediate vicinity of such structures (Figure 6, $A$ ). Delivery of electric pulses caused an increase in tissue temperature, but the maximum tissue temperature within treated tissue remained $<50^{\circ} \mathrm{C}$ (Figure 6, $B$ ). The maximum predicted temperature was reached at the end of pulse delivery and the total increase in tissue temperature was $<10^{\circ} \mathrm{C}$; temperatures returned to physiologic levels rapidly after that timepoint (Figure 6, $D-F$ ). Changes in tissue temperature estimated by simulations matched measurements from ex vivo experiments (Figure 6, C).

\section{Endobronchial Catheter-Based Electroporation of Normal Swine Lung Is Feasible and Reproducible}

Endobronchial electroporation was successfully performed in all animals, where the mean diameter of the bronchus at the location of basket placement was $6.0 \pm 1.1 \mathrm{~mm}$ (range: 4.0-7.4 mm). On CT images, the mean maximum length and long-axis and short-axis radius of tissue 

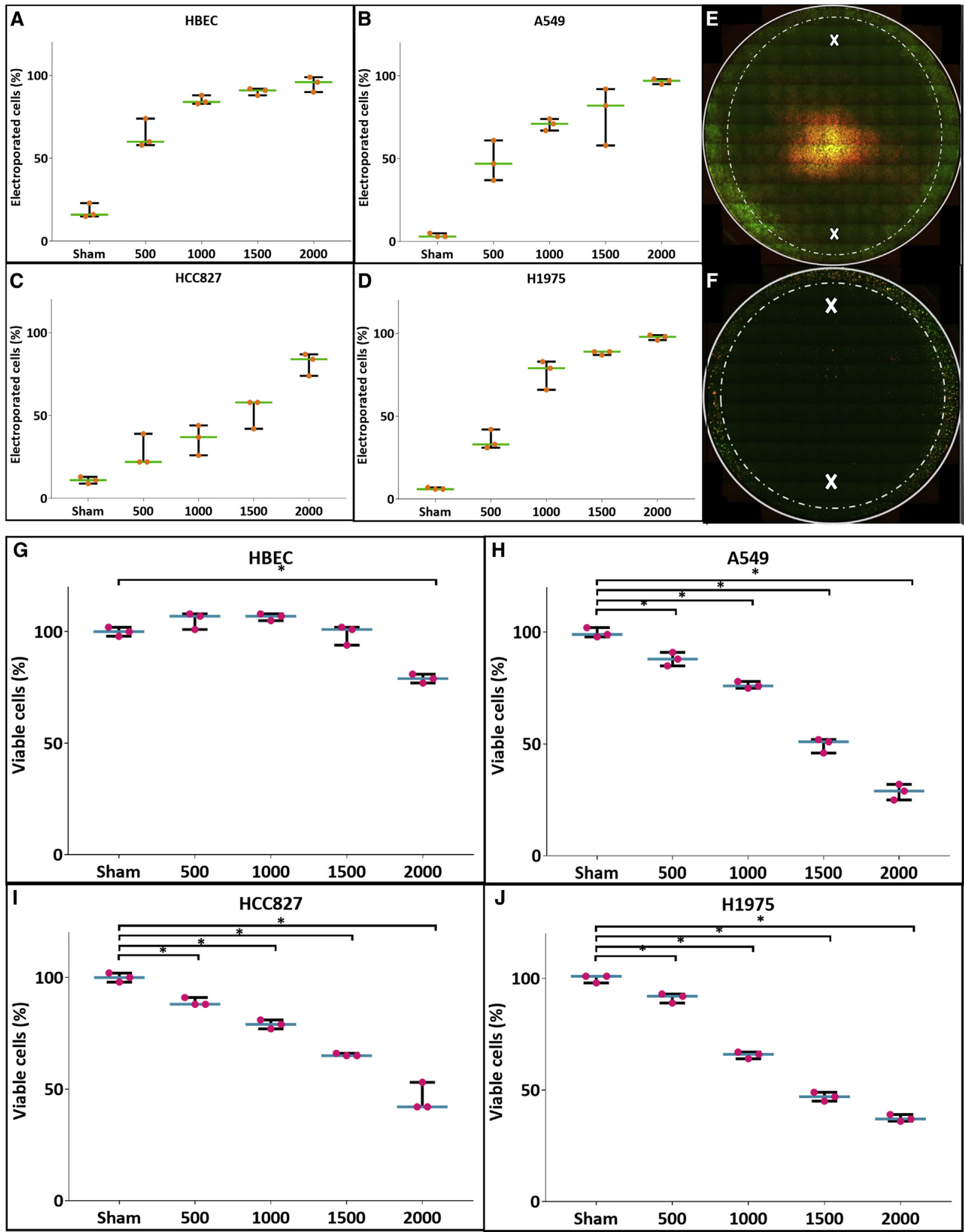

FIGURE 3. Cell viability and proliferation after in vitro electroporation. The proportion of cells that were not electroporated, either reversibly or irreversibly electroporated, and were dead were estimated by the use of propidium iodide/acridine orange staining for normal (A) and cancer cells lines (B-D). E-F, Well plates scanned after electroporation show viable cells (green fluorescence) outside the region of electroporation (marked by the dashed line). In some cells (E), the region between electrodes that experiences reduced electric field strengths undergoes reversible electroporation without complete cell death (red staining). All cells were treated at $2000 \mathrm{~V}$; the location of the electrodes is marked by an X. G-J, Cell proliferation and viability 4 hours after electroporation. $P$ values less than .05 are indicated by a bar and asterisk. HBEC, Human bronchial epithelial cell line. 


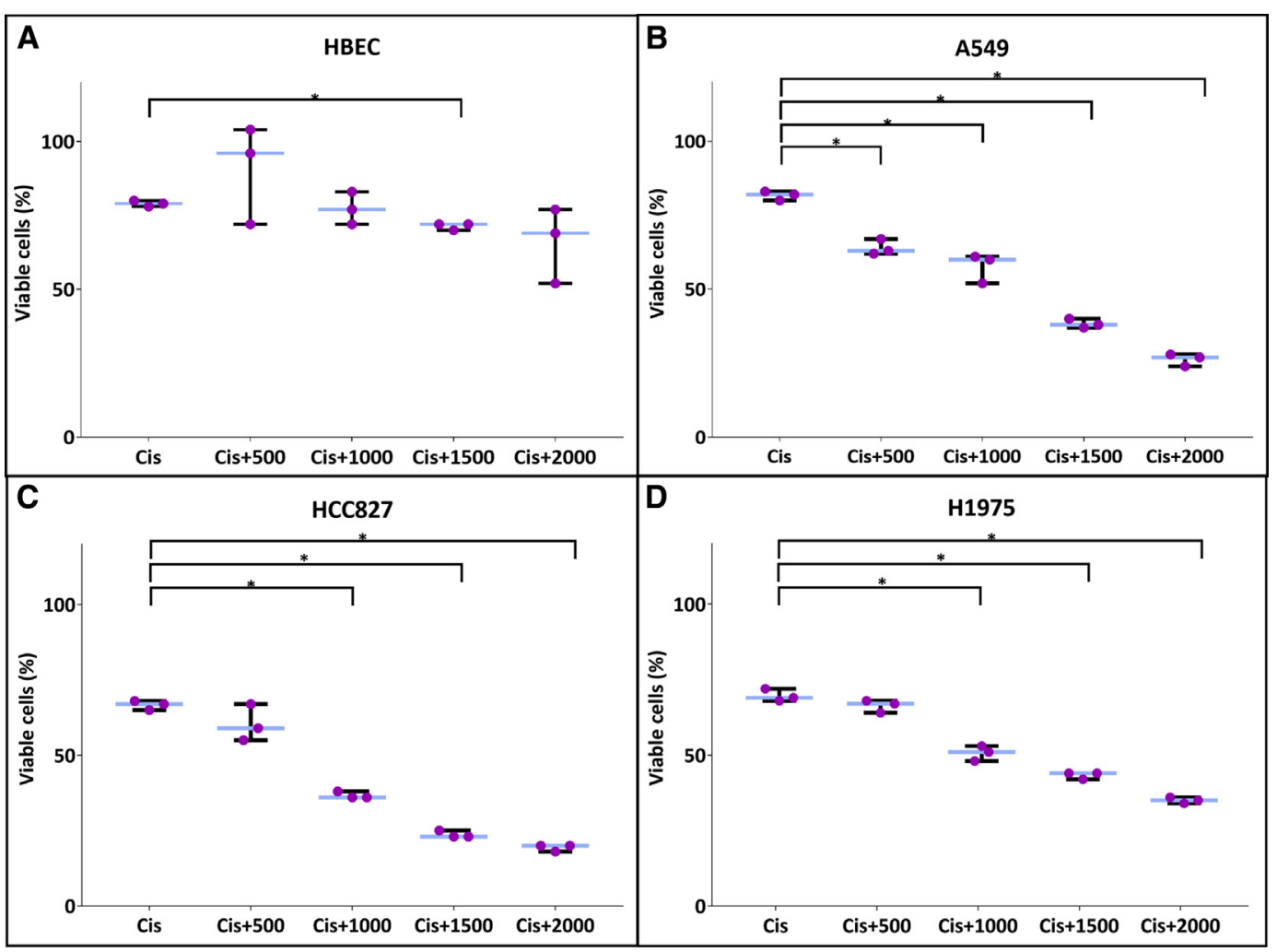

FIGURE 4. Results of combination treatment with electroporation and cisplatin. Cell proliferation at 24 hours after electroporation in the presence of cisplatin compared with treatment with drug alone at $\mathrm{IC}_{50}$ values for (A) normal and (B-D) cancer cell lines. $P$ values less than .05 are indicated by a bar and asterisk. HBEC, Human bronchial epithelial cell line.

demonstrating treatment-related changes were $48.9 \pm 4.9 \mathrm{~mm}$ (range: $40.0-55.4 \mathrm{~mm}$ ), $14.4 \pm 1.4 \mathrm{~mm}$ (range: $12.8-17.1 \mathrm{~mm}$ ), and $10.5 \pm 1.3 \mathrm{~mm}$ (range: 9.2$13.4 \mathrm{~mm}$ ), respectively. On gross pathology, mean maximum length of tissue demonstrating treatmentrelated changes along the airway tract was $24.7 \pm 3.3 \mathrm{~mm}$ (range: $20.5-29.3 \mathrm{~mm}$ ), which was significantly lesser than that of corresponding measurement on CT imaging $(P=.004)$. Mean long-axis and short-axis radius measurements on gross pathology slice were $17.2 \pm 3.0 \mathrm{~mm}$ (range: $14.5-20.5$ ) and $8.8 \pm 0.6 \mathrm{~mm}$ (range: 8.2-9.2) respectively, and these were not significantly different from corresponding measurements on $\mathrm{CT}$ imaging. The long-axis and short-axis measurements of electroporation-related changes on both $\mathrm{CT}$ and gross pathology were representative of values predicted using simulations $(P<.001)$. On H\&E staining, all specimens collected 4 hours after electroporation demonstrated
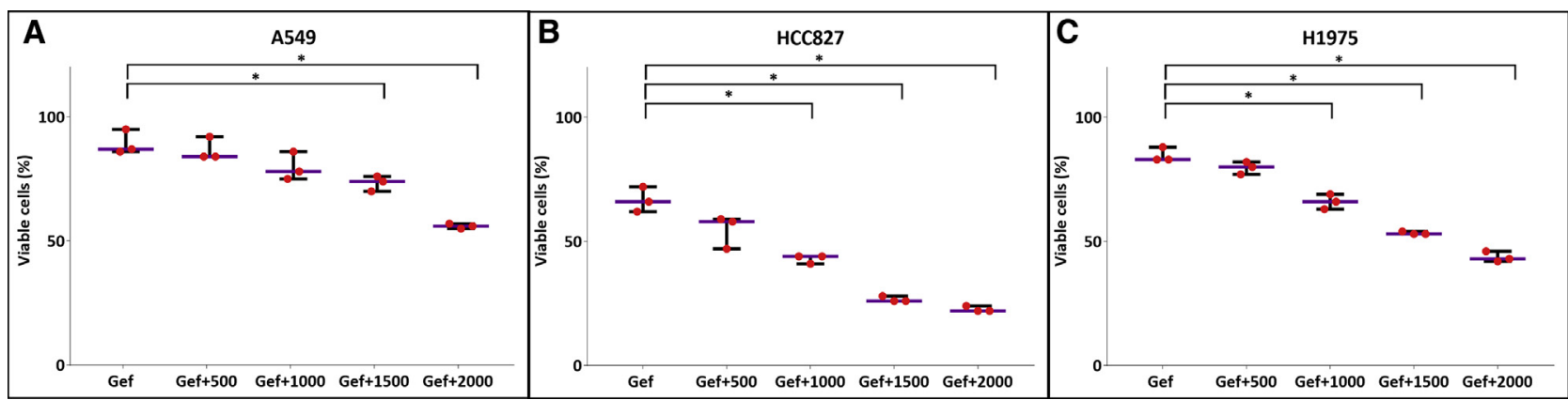

FIGURE 5. Results of combination treatment with electroporation and gefitinib. Cell proliferation at 24 hours after electroporation in the presence of gefitinib compared with treatment with drug alone at $\mathrm{IC}_{50}$ values for $(\mathrm{A}-\mathrm{C})$ cancer cell lines is shown. 

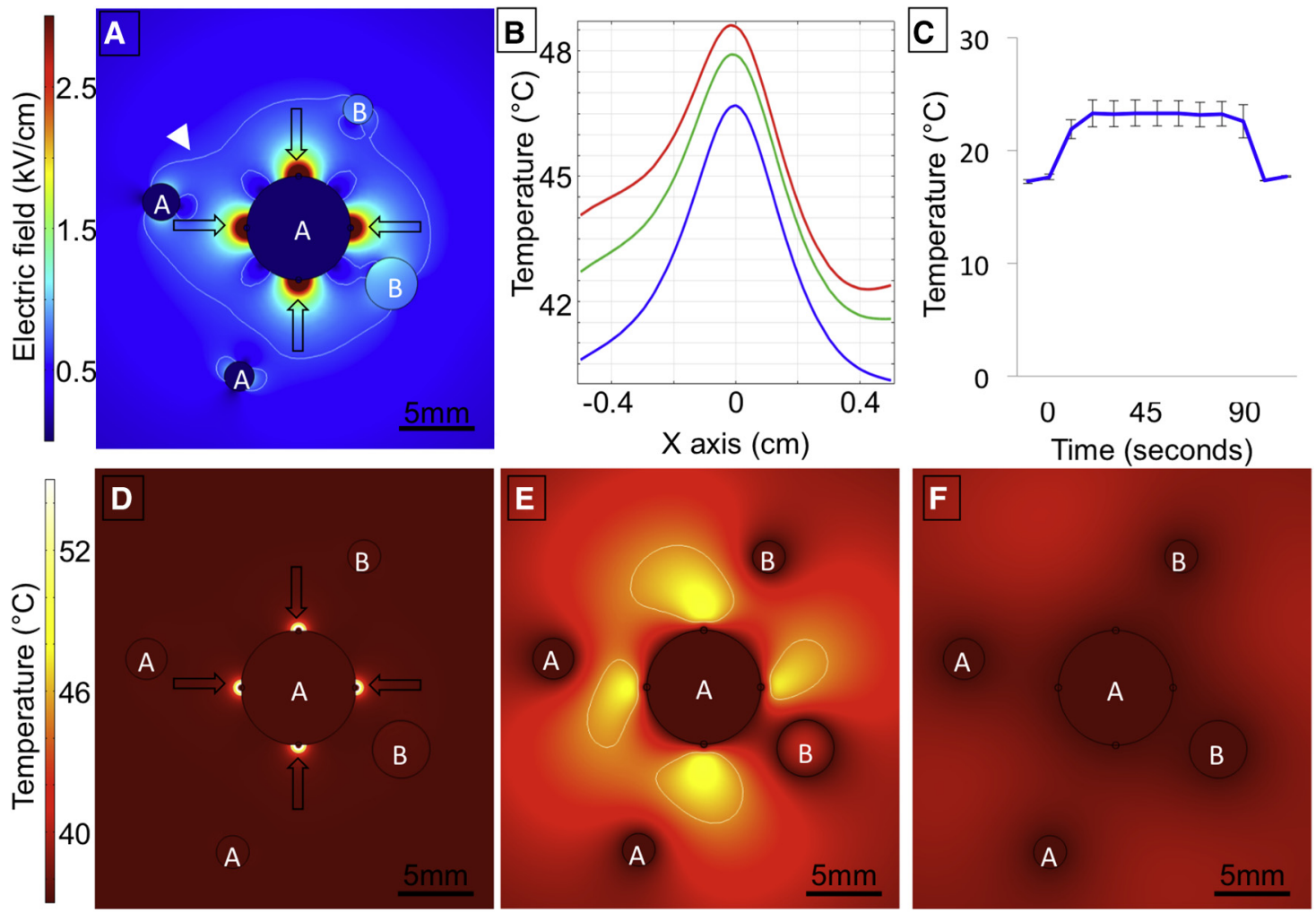

FIGURE 6. Electric field distribution and treatment-related temperature changes estimated with numerical simulations. A, Electric field distribution during pulse delivery with endobronchial basket electrode (arrows). The $500 \mathrm{~V} / \mathrm{cm}$ field strength, which is the lower bound for electroporation, is demarcated by a white line (arrowhead). B, Tissue temperature expected at the immediate vicinity of one of the electrodes. Blue, red, and green lines represent temperature distribution at immediately before pulse delivery, at the end of the last pulse, and at 80 seconds after all pulses were delivered, respectively. C, Ex vivo temperature measurement adjacent to the electrode when pulses were delivered at 2000 V. Tissue heat maps: (D) At the beginning of the first pulse, electrode locations are marked by arrows; (E) At the end of pulse delivery; and (F) 80 seconds after all pulses were delivered.

sloughing of bronchial epithelium, transmural hemorrhage, hyperemic congestion, and necrosis of submucosal glands. There was increased presence of inflammatory cells and interstitial edema in the parenchyma (Figure 7). Compared with sham-treated specimens, there was prominent terminal deoxynucleotidyl transferase-mediated dUTP nick end labeling staining in cell nuclei of the bronchial wall but staining was moderate in the peribronchial parenchyma. Anticleaved caspase-3 antibody-stained specimens showed diffuse apoptotic cells in the treated bronchi and prominent presence of apoptotic cells in the treated parenchyma.

\section{Endobronchial Electroporation Is Marked by Rapid Recovery of Lung Parenchyma from Treatment Effects}

The mean distance between the basket electrode and the heart was $4.9 \pm 1.2 \mathrm{~cm}$ (range, $3.0-7.0 \mathrm{~cm}$ ) and electric pulse delivery did not cause intraprocedural adverse effects such as arrhythmia or tachycardia. Immediate postelectroporation spasm of the bronchus (5/11 treatments) or the pulmonary vein adjacent to the bronchus (3/11 treatments) was observed on CT. There was significant reduction in the diameter of the bronchi (Figure 2, $C$ and $D$ ) from baseline to a mean value of $3.8 \pm 0.3 \mathrm{~mm}$ (range, $3.3-4.3 \mathrm{~mm}$; $P<.001)$ and pulmonary veins reduced from $8.5 \pm 0.4 \mathrm{~mm}$ (range, $8.0-9.0 \mathrm{~mm}$ ) to $3.2 \pm 0.7 \mathrm{~mm}$ (range, $2.8-4.2 \mathrm{~mm} ; P<.001)$. There were no remarkable changes in the patency of the pulmonary artery, and incidence of oxygen desaturation, reduction in vital signs, or thrombus formation was not observed (Figure 2, E). Rapid recovery of bronchial and venous luminal diameter was seen on CT imaging performed 2 to 4 hours after treatment, but complete recovery was not observed in animals killed 4 hours postelectroporation.

A one-way repeated-measures analysis of variance showed that on longitudinal analysis IRE induced statistically significant reduction in mean bronchial $\mathrm{F}(2$, $13)=4.21, P=.038$ and pulmonary vein diameter, $\mathrm{F}(2$, 13) $=4.15, P=.04$ in acute animals, but this was not evident in the 2 animals that survived. Examination of gross pathology specimens did not reveal obvious narrowing of pulmonary vein or bronchus on visual examination, and assessment of the treated airway tract was not suggestive of thermal injury (Figure E1). The diameter of the treated 


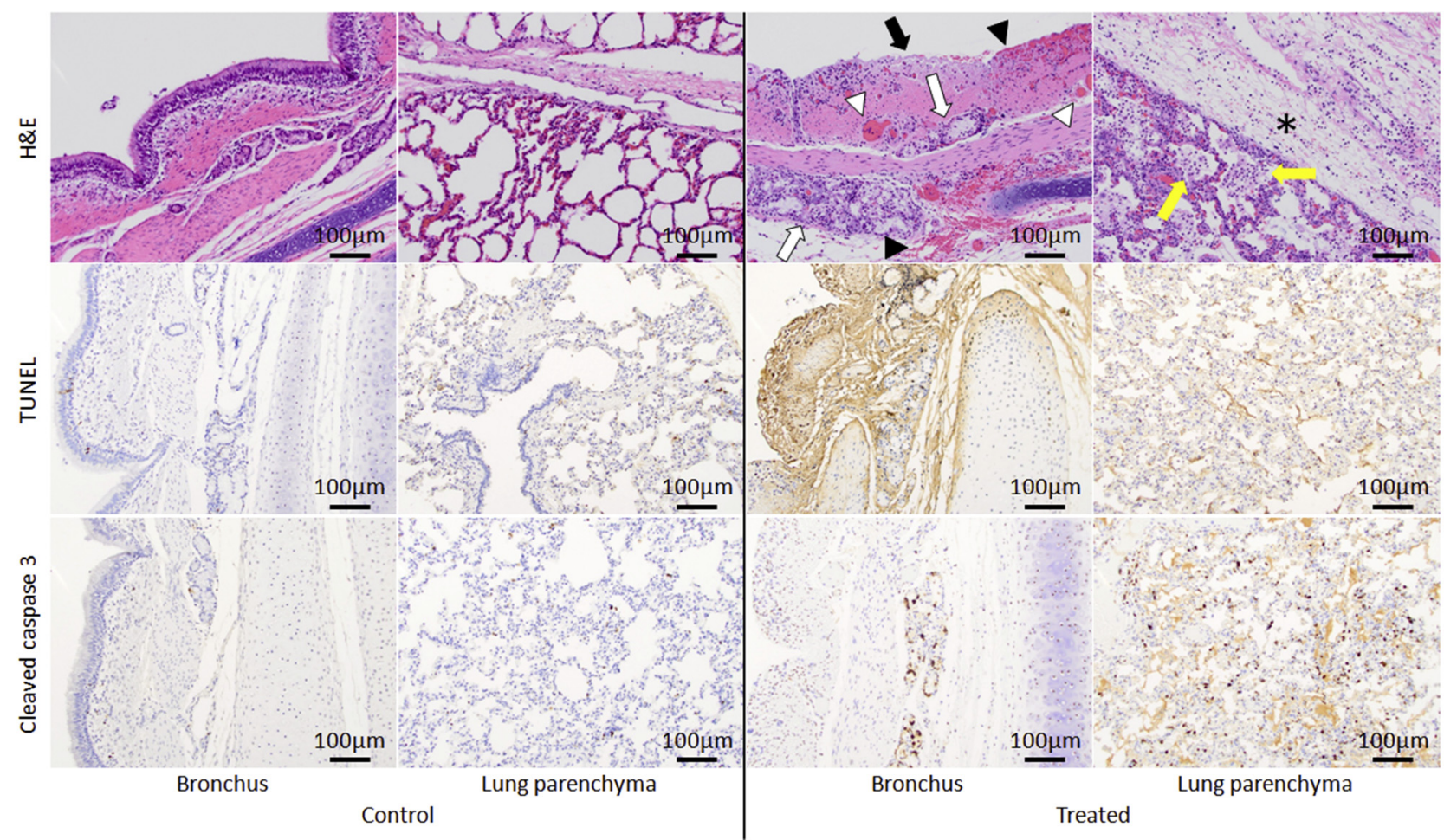

FIGURE 7. H\&E, TUNEL, and anticleaved caspase- 3 antibody-stained tissue specimens. Top row, H\&E images demonstrate sloughing of bronchial epithelium (black arrow), hemorrhage (black arrowhead), hyperemic congestion (white arrowheads), and necrosis of submucosal glands (white arrows) in electroporation-treated bronchus but not in the sham-treated sample. Peribronchial parenchyma demonstrates increased presence of inflammatory cells (yellow arrow) and edema. Middle row, There is increased and prominent TUNEL staining in the bronchial wall and moderate staining in the lung parenchyma, indicating cell death in these regions. Staining is mild or absent in the sham-treated samples. Bottom row, There is widespread staining for cleaved caspase 3 in cell nuclei in the treated parenchyma and moderate staining in the bronchial wall, indicating cells in various stages of apoptosis. Staining is mild or absent in the sham-treated samples. All images taken at $20 \times$ magnification. $H \& E$, Hematoxylin and eosin; TUNEL, terminal deoxynucleotidyl transferase-mediated dUTP nick end labeling.

bronchi and pulmonary veins within the treatment region was no different from baseline on CT imaging performed at day 7 in both animals. The treatment zone resolved rapidly, revealing minimal treatment-related changes on day $7 \mathrm{CT}$ imaging. CT imaging findings of the treatment region on day 28 were not different from untreated lung (Figure 2, $F$ and $G$ ). Clinical monitoring of 2 animals over the 28-day period was unremarkable, without any treatment-related adverse effects or complications. H\&Estained samples from the animals killed 28 days posttreatment did not yield evidence of granulation tissue or remodeling of the treated airway.

\section{DISCUSSION}

Patients with malignant central airway obstruction usually cannot undergo surgical treatment because of unfavorable tumor location or poor baseline cardiovascular function. ${ }^{1}$ Instead, bronchoscopy-guided interventions are performed, often in an emergency setting, to stabilize the patient and restore airway patency with stenting. ${ }^{9-13}$ However, debulking of the peribronchial tumor is crucial to ensure continued stent patency, without which additional interventions are required to maintain lung function. ${ }^{14-16}$ The results of this study support endobronchial electroporation as a new option for the debulking of peribronchial tumor without causing adverse events or affecting airway patency, which may also prolong the obstruction-free lifetime of stents.

Within this study, the basket of the catheter was unfurled at a location that was at least $3 \mathrm{~cm}$ from the heart. Nonetheless, Deodhar and colleagues ${ }^{23}$ examined the effect of IRE in swine lung and myocardium and concluded that unsynchronized IRE close to the heart $(<1.7 \mathrm{~cm})$ can lead to lethal ventricular arrhythmias. However, synchronization of electric pulse delivery with the absolute refractory period of the heart circumvents fatal arrhythmias. These results implicate that electric pulse delivery for treating bronchial tumors near the heart can be performed with the use of electrocardiogram gating.

The simulation models and in vivo results indicate that tissue in the vicinity of the basket electrode experiences stronger electric fields that induce cell death from IRE, 
which can allow ablation of tumor infiltrating the bronchus. Simultaneously, tissue further away from the electrodes will undergo RE, where combination with intravenously administered cisplatin may be required for tumor destruction. Together, these 2 effects can be used to destroy tumors surrounding the bronchus until depths of 1 to $2 \mathrm{~cm}$. Further refinements of the device and optimization of pulse parameters can support treatment of larger tumors.

Electroporation supports penetrative treatment of the peribronchial lung without substantial changes in tissue temperature that is common to techniques such as electrocautery, laser ablation, and radiofrequency ablation. Unlike these thermal ablation techniques, treatment penetration with electroporation is not affected by changes in treated tissue, such as bleeding, which has been reported to interfere with laser ablation. ${ }^{3,4}$ The quantum of energy deposited into the tissue during electroporation is substantially lower $(<1 \mathrm{~kJ})$ compared with laser ablation or electrocautery, which may limit the incidence of airway perforation during treatment. Electroporation has been widely reported to leave extracellular matrix components and collagenous structures within the treatment zone unaffected, ${ }^{24,25}$ which may underlie the preservation of airway patency in study animals even without stent placement. The preservation of the extracellular matrix may also have aided the rapid recovery of the treated lung parenchyma, but the exact mechanisms are not clear at this point. Because IRE is a nonionizing technique based on a nonthermal working mechanism, repeated treatment is potentially feasible, making it an attractive option for patients with recurrent disease or tumor infiltration into the bronchus. Unlike existing ablation techniques, preprocedural numerical simulations can accurately predict the size and shape of tissue that will undergo electroporation, and such planned treatment delivery can enhance the safety of this technique. The test device is well suited for fluoroscopyguided treatment of the central airways, which may be useful for the management of patients in whom bronchoscopy can exacerbate airway symptoms. Transient bronchial and venous spasm was the only treatment-related adverse event observed after electroporation. Electric pulses used for electroporation can electrically stimulate smooth and skeletal muscle ${ }^{26}$ which can explain both the incidence of spasm as well as its transient nature. ${ }^{27}$

Results from in vitro experiments of this study provide strong evidence to support combining electroporation with drugs such as cisplatin and gefitinib, which are commonly used for the adjuvant therapy of lung cancer. Although electroporation in the presence of either drug significantly increased cell death, such effects were most prominent in cells treated at lower voltages. These results were concordant with incidence of RE at lower voltages, where cells would recover viability in the absence of cytotoxic drugs and direct cytotoxic effect of IRE at greater voltages. Transient permeabilization of the cell membrane permits free diffusion of drugs into the cell, and this effect especially benefits drugs that undergo receptor or transporter-mediated entry into the cytoplasm. Cisplatin uptake in cells is understood to be coordinated by the copper transporter Ctrlp. ${ }^{28}$ Electroporation may support uptake of cisplatin while bypassing the transporter and can assist treatment of tumors that develop resistance to the drug because of mutations or deletion of the Ctrl gene. Interestingly, combination therapy with electroporation increased the therapeutic effect of gefitinib in both sensitive (HCC827) and insensitive cell lines (H1975). The mechanisms underlying increased effectiveness of this combination therapy is not clear and requires further exploration.

There are several limitations in our study. There are no validated large animal models of lung cancer that impeded testing the efficacy of the device and electroporation in vivo. Because this is the first study investigating endobronchial catheter-based electroporation for lung cancer, it was not possible to determine a precise sample size. Instead, comparable studies investigating ablation in endoluminal setting in a porcine model were used to guide the selection of animal numbers and experimental design. It is expected that tumor clearance after electroporation will not be similar to regeneration of normal lung reported in this study, and the risk of complications such as fistula formation is unknown. The electrical conductance and thermal properties of lung tumors vary substantially from normal lung, and this may affect the shape and size of the tissue that undergoes electroporation. The diameter of our device is larger than the working channel of bronchoscopes, and further refinement of the device will be required before bronchoscopy-guided ablation in patients. Pilot human studies may be required to answer some of these questions and also to validate the preclinical findings of this study.

\section{CONCLUSIONS}

Catheter-based endobronchial electroporation is a reproducible technique that can be used to treat peribronchial tumors in combination with cisplatin or gefitinib. Electroporation does not affect the patency of the treated bronchus or lung and can be developed for the treatment of malignant central airway obstructions.

\section{Conflict of Interest Statement}

Authors have nothing to disclose with regard to commercial support.

\section{References}

1. Ernst A, Feller-Kopman D, Becker HD, Mehta AC. Central airway obstruction. Am J Respir Crit Care Med. 2004;169:1278-97.

2. Wahidi MM, Unroe MA, Adlakha N, Beyea M, Shofer SL. The use of electrocautery as the primary ablation modality for malignant and benign airway obstruction. J Thorac Oncol. 2011;6:1516-20. 
3. Gesierich W, Reichenberger F, Fertl A, Haeussinger K, Sroka R. Endobronchial therapy with a thulium fiber laser (1940 nm). J Thorac Cardiovasc Surg. 2014; 147:1827-32.

4. Mantovani G, Astara G, Manca G, Versace R, Contu P, Carai A. Endoscopic laser ablation as palliative treatment of endobronchial, nonresectable, or recurrent lung cancer: assessment of its impact on quality of life. Clin Lung Cancer. 2000;1: 277-85.

5. Taber SW, Buschemeyer WC III, Fingar VH, Wieman TJ. The treatment of malignant endobronchial obstruction with laser ablation. Surgery. 1999;126:730-3.

6. Tsushima K, Koizumi T, Tanabe T, Nakagawa R, Yoshikawa S, Yasuo M, et al. Bronchoscopy-guided radiofrequency ablation as a potential novel therapeutic tool. Eur Respir J. 2007;29:1193-200.

7. Koizumi T, Tsushima K, Tanabe T, Agatsuma T, Yokoyama T, Ito M, et al. Bronchoscopy-guided cooled radiofrequency ablation as a novel intervention therapy for peripheral lung cancer. Respiration. 2015;90:47-55.

8. Schumann C, Hetzel M, Babiak AJ, Hetzel J, Merk T, Wibmer T, et al. Endobronchial tumor debulking with a flexible cryoprobe for immediate treatment of malignant stenosis. J Thorac Cardiovasc Surg. 2010;139:997-1000.

9. Stephens KE Jr, Wood DE. Bronchoscopic management of central airway obstruction. J Thorac Cardiovasc Surg. 2000;119:289-96.

10. Mahmood K, Wahidi MM, Thomas S, Argento AC, Ninan NA, Smathers EC, et al. Therapeutic bronchoscopy improves spirometry, quality of life, and survival in central airway obstruction. Respiration. 2015;89:404-13.

11. Jeon K, Kim H, Yu CM, Koh WJ, Suh GY, Chung MP, et al. Rigid bronchoscopic intervention in patients with respiratory failure caused by malignant central airway obstruction. J Thorac Oncol. 2006;1:319-23.

12. Neuberger M, Hapfelmeier A, Schmidt M, Gesierich W, Reichenberger F, Morresi-Hauf A, et al. Carcinoid tumours of the lung and the 'PEPPS' approach: evaluation of preoperative bronchoscopic tumour debulking as preparation for subsequent parenchyma-sparing surgery. BMJ Open Respir Res. 2015;2: e000090.

13. Lin CY, Chung FT. Central airway tumors: interventional bronchoscopy in diagnosis and management. J Thorac Dis. 2016;8:E1168-76.

14. Al-Ayoubi AM, Bhora FY. Current readings: the role of stenting in tracheobronchial disease. Semin Thorac Cardiovasc Surg. 2014;26:71-5.

15. Grosu HB, Eapen GA, Morice RC, Jimenez CA, Casal RF, Almeida FA, et al. Stents are associated with increased risk of respiratory infections in patients undergoing airway interventions for malignant airways disease. Chest. 2013;144: 441-9.

16. Ost DE, Ernst A, Grosu HB, Lei X, Diaz-Mendoza J, Slade M, et al. Complications following therapeutic bronchoscopy for malignant central airway obstruction: results of the AQuIRE Registry. Chest. 2015;148:450-71.

17. Weaver JC. Electroporation theory. Concepts and mechanisms (review). Methods Mol Biol. 1995;55:3-28.
18. Silk M, Tahour D, Srimathveeravalli G, Solomon SB, Thornton RH. The state of irreversible electroporation in interventional oncology. Semin Intervent Radiol. 2014;31:111-7.

19. Silk MT, Wimmer T, Lee KS, Srimathveeravalli G, Brown KT, Kingham PT, et al Percutaneous ablation of peribiliary tumors with irreversible electroporation. $J$ Vasc Interv Radiol. 2014;25:112-8.

20. Yang W, Soares J, Greninger P, Edelman EJ, Lightfoot H, Forbes S, et al. Genomics of Drug Sensitivity in Cancer (GDSC): a resource for therapeutic biomarker discovery in cancer cells. Nucleic Acids Res. 2013;41(Database issue):D955-61.

21. Hasgall PA, Di Gennaro F, Baumgartner C, Neufeld E, Gosselin MC, Payne D et al. IT'IS Database for thermal and electromagnetic parameters of biological tissues, version 3.0. September 1, 2015. Available at: https://www.itis.ethz.ch/ virtual-population/tissue-properties/overview/. Accessed January 9, 2018.

22. Neal RE II, Garcia PA, Robertson JL, Davalos RV. Experimental characterization and numerical modeling of tissue electrical conductivity during pulsed electric fields for irreversible electroporation treatment planning. IEEE Trans Biomed Eng. 2012;59:1076-85.

23. Deodhar A, Dickfeld T, Single GW, Hamilton WC Jr, Thornton RH, Sofocleous CT, et al. Irreversible electroporation near the heart: ventricular arrhythmias can be prevented with ECG synchronization. AJR Am J Roentgenol. 2011;196:W330-5

24. Srimathveeravalli G, Silk M, Wimmer T, Monette S, Kimm S, Maybody M, et al. Feasibility of catheter-directed intraluminal irreversible electroporation of porcine ureter and acute outcomes in response to increasing energy delivery. $J$ Vasc Interv Radiol. 2015;26:1059-66.

25. Srimathveeravalli G, Cornelis F, Wimmer T, Monette S, Kimm SY, Maybody M, et al. Normal porcine ureter retains lumen wall integrity but not patency following catheter-directed irreversible electroporation: imaging and histologic assessment over 28 days. $J$ Vasc Interv Radiol. 2017;28:913-9.e1.

26. Arena CB, Sano MB, Rossmeisl JH Jr, Caldwell JL, Garcia PA, Rylander MN, et al. High-frequency irreversible electroporation (H-FIRE) for non-thermal ablation without muscle contraction. Biomed Eng Online. 2011;10:102.

27. Narayanan G, Bhatia S, Echenique A, Suthar R, Barbery K, Yrizarry J. Vessel patency post irreversible electroporation. Cardiovasc Intervent Radiol. 2014; 37:1523-9.

28. Ishida S, Lee J, Thiele DJ, Herskowitz I. Uptake of the anticancer drug cisplatin mediated by the copper transporter Ctr1 in yeast and mammals. Proc Natl Acad Sci U S A. 2002;99:14298-302.

Key Words: central airway obstruction, catheter based therapy, electroporation, drug delivery, lung cancer 


\section{APPENDIX E1. CULTURE METHODS AND TREATMENT DETAILS \\ Culture Methods}

The cancer cells were grown in Roswell Park Memorial Institute culture medium (supplemented with 10\% fetal calf serum, penicillin, and streptomycin), and the human bronchial epithelial cell line was grown in keratinocyte serum-free media (supplemented with epidermal growth factor and bovine pituitary extract). All cells were maintained in a $\mathrm{CO}_{2}$-humidified incubator at $37^{\circ} \mathrm{C}$ until they reached $80 \%$ confluence, after which they were harvested and seeded on to 24-well plates.

\section{Electroporation Details}

All cells were treated with pulses $(70$ pulses at $1 \mathrm{~Hz}, 90-$ $\mu$ s pulse length) delivered at varying voltages (500, 1000, 1500 , or $2000 \mathrm{~V}$ ) using an ECM 830 pulse generator (BTX, Holliston, Mass). Electric pulses were delivered to the cells via 2 stainless-steel pin electrodes $(25-\mathrm{mm}$ long, 1 -mm width) that were kept parallel at 10 -mm spacing.

\section{Chemotherapy Details}

Cisplatin and gefitinib were both dissolved in eimethyl sulfoxide and titrated to obtain drug concentrations at $\mathrm{IC}_{50}$ values (cisplatin: H1975 - 5.53 mM; HCC827 - 8.29 mM; A549 - $10.9 \mathrm{mM}$; HBEC - $10.9 \mathrm{mM}$; and gefitinib: H1975 - 9.07 mM; HCC827 - 0.0198 mM; A549 - 1.96 mM) reported in the Genomics of Drug Sensitivity in Cancer Web site. ${ }^{20}$ Cisplatin or gefitinib was added to cells in 24-well plates and were electroporated via the parameters described previously or held as drug only control.

\section{APPENDIX E2. DESIGN AND CONSTRUCTION OF ENDOBRONCHIAL ELECTROPORATION CATHETER}

A multilumen catheter $(90 \mathrm{~cm}, 9 \mathrm{Fr})$ was custom manufactured (Vention Medical, Plainfield, NJ) with a central lumen for 0.038 in guidewire access surrounded by 4 smaller peripheral channels (0.02 in). Nitinol wire (0.012 in diameter, $210 \mathrm{~cm}$; McMaster-Carr Inc, Robbinsville, $\mathrm{NJ}$ ) was threaded through the peripheral lumen to construct a basket electrode at the tip of the catheter (Figure 1, $A$ and $B$ ). Inserting and withdrawing the wires at the catheter hub expanded or collapsed the basket electrode and supporting electrical contact within lumen of varying diameter (0.3$3.5 \mathrm{~cm}$ ) (Figure 1, $C$ and $F$ ).

\section{APPENDIX E3. DETAILS ON NUMERICAL MODELS}

A 2-dimensional geometric mesh representing a (1) mainstem bronchus, (2) the basket electrode deployed within the bronchus, and (3) the surrounding lung parenchyma with additional airways and blood vessels was created via Comsol Multiphysics software (Comsol Inc, Los Altos, Calif). Electrical and mechanical properties of inflated lung described by Hasgall and colleagues ${ }^{21}$ were assigned to model. The Laplace equation incorporating dynamic changes in the electrical conductivity of the tissue because of electroporation as described by Neal and colleagues ${ }^{22}$ was solved to determine electric field distribution from pulses of varying voltages $(500-2000 \mathrm{~V})$ and fixedpulse parameters $(90-\mu$ s pulse length, 70 pulses delivered at $1 \mathrm{~Hz}$ ). The simulation was coupled with the Pennes Bioheat equation to estimate changes in tissue temperature from pulse application. Simulation covered a total time of 150 seconds, which included the duration of pulse delivery (70 seconds) and a posttreatment refractory period of 80 seconds.

\section{APPENDIX E4. EX VIVO TEMPERATURE MEASUREMENT PROCEDURE}

Normal porcine lung was harvested for ex vivo temperature measurements. Electric pulse delivery was performed between the basket electrode placed into the mainstem bronchus and a grounding pad placed on the surface of the inflated lung. A fiberoptic thermometer (LumaSense Technologies Inc, Santa Clara, Calif) was placed immediately adjacent to the basket electrode, and temperature was recorded during pulse delivery. Temperature measurements were repeated in triplicate during electroporation at $2000 \mathrm{~V}$.

\section{APPENDIX E5. IMAGING ANALYSIS}

The effects of reversible electroporation and irreversible electroporation can manifest as regions of lung parenchyma demonstrating consolidation and hemorrhage on computed tomography imaging. For each treatment region, the maximum length (along the airway), and the long-axis and short-axis radius (perpendicular to the airway) were measured. In addition, the minimum distance between treated bronchus and heart, the internal diameter of treated bronchus, and the diameter of pulmonary artery or vein adjacent to the treated bronchus was also recorded.

\section{APPENDIX E6. METHODS USED FOR GROSS PATHOLOGY AND HISTOPATHOLOGY}

The treated airway was opened longitudinally to expose its mucosal surface, and locations of electrode placement were identified. Photographs were taken for gross measurement of the extent of electroporation from the bronchus. Specimens from the center of the treatment region were fixed in $10 \%$ neutral buffered formalin, processed, embedded in paraffin, and stained with hematoxylin and eosin, terminal deoxynucleotidyl transferase-mediated dUTP nick end labeling (cell death) and anticleaved caspase-3 (cell apoptosis). Slides were analyzed by a board-certified veterinary pathologist (S.M.) for airway 
integrity, condition of the extracellular matrix, and hemorrhage in samples from the acute timepoint. Samples acquired 28 days posttreatment were assessed for the patency of the airway, presence of granulation tissue, and signs of airway remodeling.

\section{APPENDIX E7. STATISTICAL ANALYSIS}

Simulation results and the region of treatment effects observed on computed tomography imaging and gross histopathology were compared with single-sample hypothesis testing to determine whether depth of treatment penetration from the bronchial wall was the representative outcome of experimental treatment parameters. Measurements on computed tomography and gross pathology were compared with the Student $t$ test. Paired $t$ test was used to compare pre- and posttreatment changes in lumen diameter of bronchus and pulmonary vessels that underwent electroporation. A one-way repeated-measures analysis of variance was performed to determine whether there were differences overtime in bronchus and pulmonary vein diameter due to irreversible electroporation. The Bonferroni method was used to correct for multiple comparisons, given the large number of variables used to model in vitro proliferation scores. 


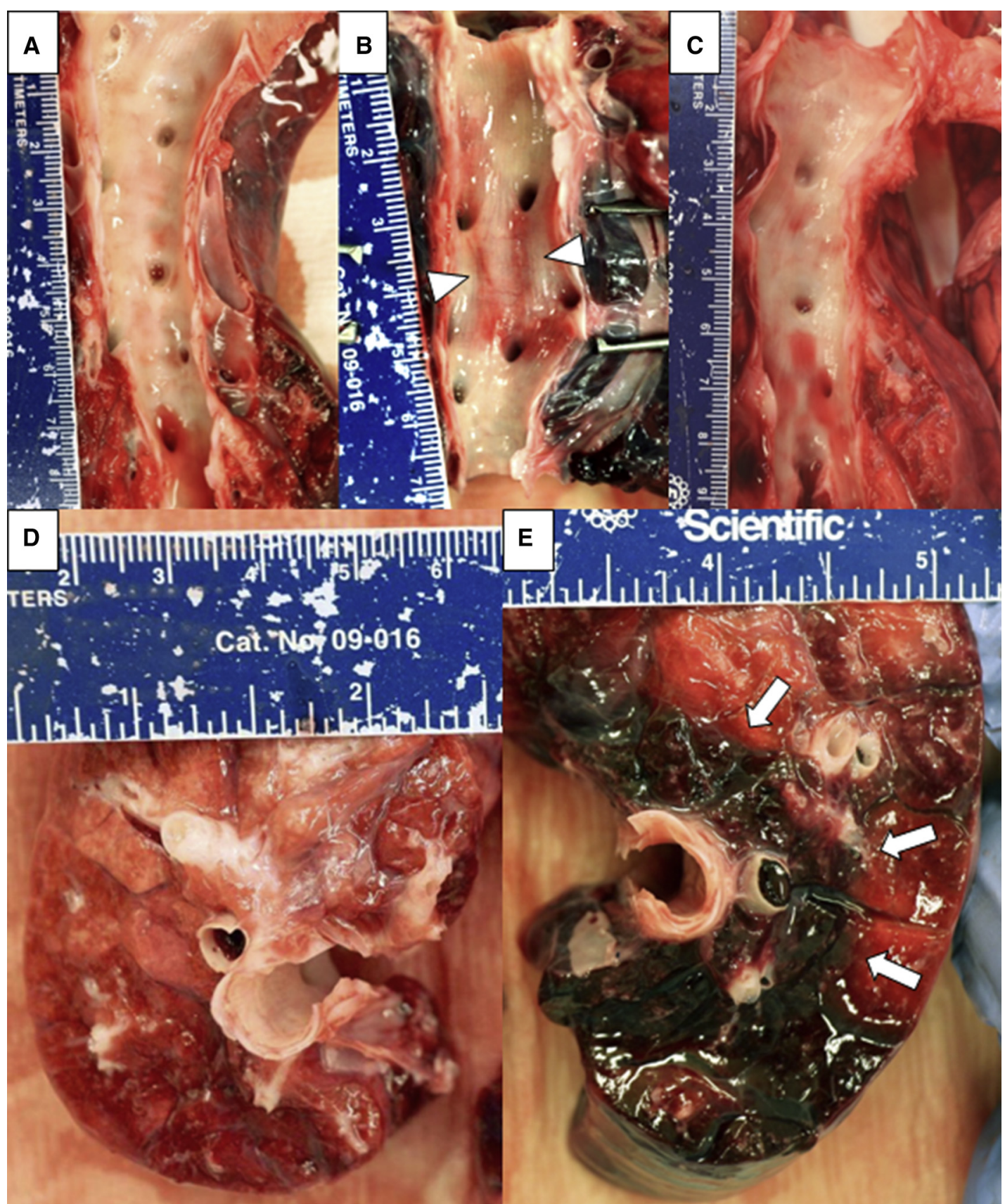

FIGURE E1. Gross appearance of electroporation-treated and sham-treated bronchus and lung. A, Sham-treated lung. B, Bronchial wall 4 hours after electroporation. The location of electrode basket placement has a distinct appearance (arrowheads). C, Bronchial wall appears unremarkable at day 28 after electroporation. Comparison of sham-treated (D) and electroporation-treated (E) lung (acute timepoint). There is evidence of consolidation and hemorrhage (arrows) in the peribronchial lung, consistent with tissue changes in tissue treated with electroporation. 